\title{
Research and Implementation of Axial 3D Printing Method for PLA Pipes
}

\author{
Haiguang Zhang ${ }^{1,2,3, * \mathbb{D}}$, Wenguang Zhong ${ }^{1} \mathbb{D}$, Qingxi Hu ${ }^{1,2,3}$, Mohamed Aburaia ${ }^{4}$, \\ Joamin Gonzalez-Gutierrez ${ }^{5}$ (D) and Herfried Lammer ${ }^{6}$ \\ 1 Rapid Manufacturing Engineering Center, School of Mechatronic Engineering and Automation, Shanghai \\ University, Shanghai 200444, China; wenguang@i.shu.edu.cn (W.Z.); huqingxi@shu.edu.cn (Q.H.) \\ 2 Shanghai Key Laboratory of Intelligent Manufacturing and Robotics, Shanghai University, \\ Shanghai 200072, China \\ 3 National Demonstration Center for Experimental Engineering Training Education, Shanghai University, \\ Shanghai 200444, China \\ 4 Competence Center Digital Manufacturing and Robotics, University of Applied Science Technikum Wien, \\ Höchstädtplatz 6, 1200 Wien, Austria; aburaia@technikum-wien.at \\ 5 Institute of Polymer Processing, Montanuniversitaet Leoben, Otto Gloeckel-Strasse 2, 8700 Leoben, Austria; \\ joamin.gonzalez-gutierrez@unileoben.ac.at \\ 6 Kompetenzzentrum Holz GmbH, Altenberger Straße 69, 4040 Linz, Austria; h.lammer@wood-kplus.at \\ * Correspondence: haiguang_zhang@i.shu.edu.cn; Tel.: +86-158-2126-4716
}

Received: 19 June 2020; Accepted: 5 July 2020; Published: 7 July 2020

check for updates

\begin{abstract}
Additive manufacturing has been applied in many fields, but its layer-by-layer fabrication process leads to a weak inter-layer bond strength of printed parts, so it cannot meet the higher requirements for mechanical properties of the industry. At present, many researchers are studying the printing path planning method to improve the mechanical properties of printed parts. This paper proposes a method to plan the printing path according to the actual stress of pipe parts, and introduces the realization process of an algorithm in detail, and obtains the printing control G-code. Additionally, a 5-axis material extrusion platform was built to realize the printing of polylactic acid pipes with plane and space skeleton curves, respectively, which verified the feasibility and applicability of the method and the correctness of the planning path with standard material extrusion filaments. Finally, the tensile and bending experiments prove that axial printing enhances the mechanical properties of pipe parts.
\end{abstract}

Keywords: 3D printing; mechanical properties reinforcement; pipe parts; axial printing; 5-axis printing

\section{Introduction}

Additive manufacturing (AM), also known as 3D printing (3DP), has many advantages, such as complex structure construction, short shaping cycle and high material utilization rate, so it has attracted the attention of many scholars worldwide. After decades of development, it has achieved many results in materials, process, and equipment. It has been widely used in many fields [1-3]. However, AM technology still has some technical limitations, mainly including layer-by-layer stacking printing process resulting in a step effect and low surface accuracy, and insufficient adhesion between layers results in low strength in the Z-axis direction. Taking the most common material extrusion (MEX) process, it is necessary to produce supporting structures when printing cantilever or hollow parts, so that the printing time and material costs are inevitably increased. For solving the above problems, researchers in various countries have carried out a lot of research work on printing path planning methods.

Typical studies in improving the surface quality of parts produced by MEX are as follows. Jin et al. [4] proposed a slicing procedure and corresponding algorithms to determine the extruder path for a theoretical 3-axis machine; their approach could preserve tiny features on the surface by increasing the number of 
sampling points around small features, and a B-spline was used to approximate the curved surfaces accurately; however, no real parts were manufactured in their study. By rechecking the path and tool orientation conditions, Isa et al. [5] proposed a 5-axis path planning method that considers the contour of a freeform surface to prevent the effect of the staircase effects on hollow and solid components. Ding et al. [6] proposed a method based on the medial axis transformation path, planning to generate deposition paths for wire and arc additive manufacturing, which can improve the quality and material efficiency of thin-walled structures. Feng et al. [7] proposed a direct slicing algorithm of the T-spline surfaces suitable for MEX, which is precise and reliable enough for practical applications. Xie et al. [8] proposed a practical B-spline based smoothing algorithm for removing sharp corners on the printing path, and the experimental results showed that the method is feasible and effective.

Typical studies in reducing or avoiding the support structure are as follows. Lee et al. [9] proposed a method for generating three-dimensional layer information using an auto-partitioning algorithm, generated a printing path that can be used for a 5-axis platform, and used structural parts with cantilever features as examples for verification, which can realize unsupported printing simply and quickly. Ding et al. [10] proposed a multi-directional slicing method based on the stereolithography (STL) format CAD model, using the decomposition-regrouping method to achieve unsupported printing, which is shown to be efficient and straightforward on various tests parts, especially for geometries with a large number of holes. Xu et al. [11] decomposed the model into support-free parts directly on the cusp-height constraint, each with its unique build direction, and ultimately eliminated the use of support by taking advantage of the 5-axis MEX platform. Wu et al. [12] proposed a five degrees of freedom wireframe printer to print arbitrary grids, developed and applied a collision-free algorithm for local minimum area motion on the edges, and completed the printing of arbitrary wireframe grids, reducing the printing time and material cost of the model. Zhao et al. [13] proposed two nonplanar slicing approaches that are a decomposition-based curved surface slicing strategy, and a transformation-based cylinder surface slicing method, and experimental results proved the support structures and layers number are reduced. Xu et al. [14] proposed a curved layer-based process planning algorithm for multi-axis printing of an arbitrary freeform dense part, which is effective and feasible in eliminating the need for supporting structures. Dai et al. [15] proposed a five degrees of freedom (DOF) 3D printing algorithm, which can quickly and efficiently calculate the possible material deposition path, so that the fabrication can be performed in a support-free way. Khan et al. [16] proposed an improved Chopper model block unsupported printing method based on the algorithm of binary space partitioning (BSP) tree generation, which can divide large complex models into advanced small models for block 3D printing, and finally, glued the individual components to realize the model without support printing. Zhao et al. [17] proposed an approach of printing overhanging structures using inclined layer printing technology, which can easily print complex models with ordinary MEX printers, without generating supporting structures.

Typical studies in improving the mechanical properties of MEX parts are as follows. Dickson et al. [18] solved the problem of non-interaction between layers of fiber-reinforced composite materials, by printing a 0/90 woven structure in one layer and carried out open-hole tensile tests. The tensile strength of the printed sample of this woven structure was $44 \%$ higher than that of the punched specimen. Sugiyama et al. [19] used a continuous carbon fiber 3D printer to manufacture sandwich structures with honeycomb, rhombus, rectangle, and circle core shapes as a single piece. Three-point bending tests showed maximum load, and flexural modulus increased as the effective density increased for all core shapes, but the rhombus core shape was the strongest. Bin et al. [20] used a $3 \mathrm{D}$ printer on a robot arm to realize multi-plane layered printing. Compared with the tensile specimen produced by a traditional 3D printer, the elastic modulus, yield strength and ultimate tensile strength of the tensile specimen in the upright printing orientation have been improved. Huang et al. [21] proposed a curved layer adaptive slicing algorithm, combining adaptive flat layer and curved layer slicing together. The three-point bending test for different thickness of the curved layer proves that the thicker the curved layer is, the better the mechanical properties are. Lim et al. [22] applied the 
surface layer printing method to the 3D printing process of concrete, and compared with the existing flat printing path; the surface strength was higher. Tam et al. [23] used a six-axis industrial robot arm to deposit the filaments along the path derived from the principal stress line and applied 2.5D surface geometry to improve the strength of the print. According to stress calculations from a finite element analysis of the components, Xia et al. [24] constructed the tool path parallel to the direction of maximum principal stress, which efficiently improves the mechanical performance of the printing specimen, but this method was only applied to the plane layer.

It can be seen that, initially, path planning algorithms had the aim to improve the surface quality of AM printed parts, and reduce or avoid support structures. However, path planning algorithms have started to be used to improve the mechanical properties of 3D printing parts, mainly by changing the printing path of flat or curved layers [18-24]. To our knowledge, no print space path method has been used to increase the mechanical performance of printed parts. Therefore, this paper aims to enhance the mechanical properties of equal-section pipes and studies the method of planning the printing path according to the actual stress of the pipes. Finally, a multi-axis MEX platform was built to achieve enhanced printing along the force direction, with standard polylactic acid (PLA) filaments. Moreover, through mechanical testing, we aim to verify the feasibility of the method and the correctness of the planned path.

\section{Printing Scheme of Pipe Parts}

\subsection{Stress Simulation Analysis of Pipe Parts}

Pipe parts are mainly subjected to tensile and bending forces in practical applications. The finite element analysis method can be used to simulate these loading situations. Figure 1 shows the simulation of pipe parts subjected to tensile forces, and Figure 2 shows the simulation of pipe parts subjected to bending forces. Using conventional MEX processes, higher stresses concentrate in the inter-layer space, and thus, when the pipes are pulled, the printed parts are easily damaged, due to the small bonding force between the layers. Due to the influence of the staircase effect, when the pipe parts are under pressure, printed pipes are prone to stress concentration at the interlayer, which may easily cause failures. Therefore, printing along the axial direction of the pipes can avoid the above effects, thereby enhancing the mechanical properties of the printed pipe.

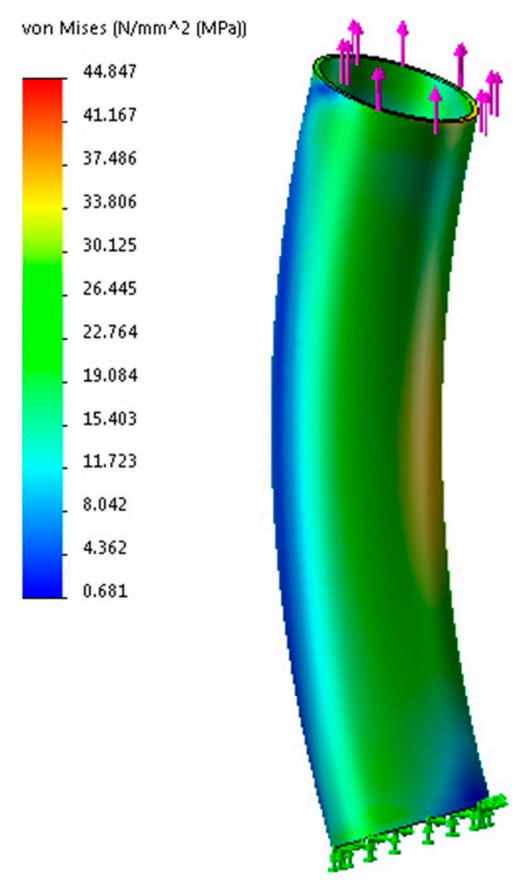

Figure 1. Tension simulation analysis. 

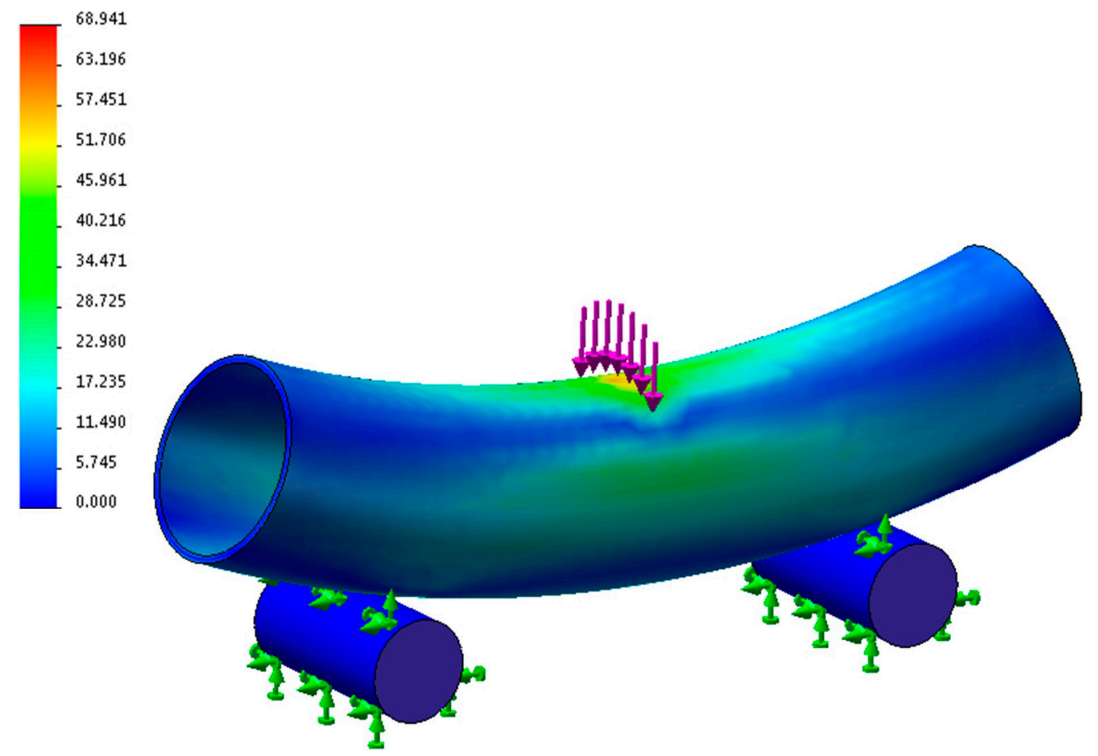

Figure 2. Three-point bending simulation analysis.

\subsection{Axial Printing Basic Scheme}

If the pipe parts are placed horizontally, the pipe parts can also be formed along the axial direction, adopting the traditional three-axis printing method. However, this method utilizes a lot of support material inside the pipe parts, inevitably resulting in material waste, difficult support removal and easy damage to the printed parts in the stripping process. To achieve both axial printing and unsupported printing, improving the freedom of the printing platform is one of the solutions. Therefore, this paper designs and develops a 5-axis MEX platform by adding the swing axis $(A$-axis) and the rotation axis (C-axis), based on the original $X / Y / Z$ moving axis. The schematic diagram of the platform structure is shown in Figure 3.

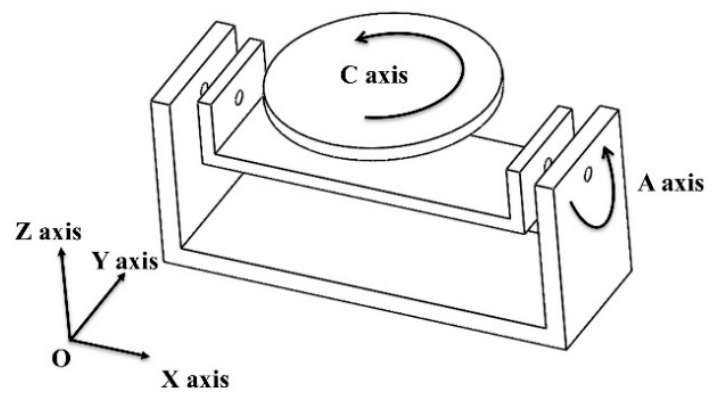

Figure 3. Schematic diagram of the 5-axis material extrusion (MEX) platform structure.

The printing process, adopting the 5-axis MEX platform, is shown in Figure 4. First, the layer-by-layer printing method is used to print a support pipe. Second, rotate $A$-axis of the platform by $90^{\circ}$ so that the support is placed horizontally. Third, the printing nozzle prints the surface path along the axis of the pipe parts. After completing one single path, the $C$-axis rotates for a certain angle, the next path is printed, and so on, until the surface of the whole pipe part is entirely printed. The rotation angle of the $C$-axis is related to printing accuracy. Finally, rotate $A$-axis by $90^{\circ}$ in the opposite direction to restore the initial position and remove the finalized pipe part. 


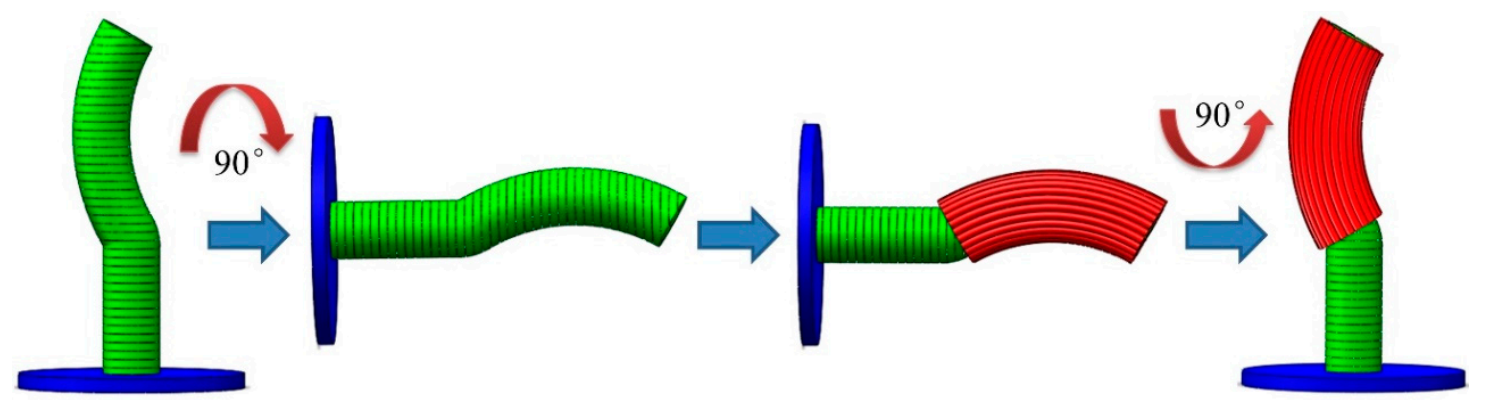

Figure 4. The printing process for axially reinforced pipes.

\section{Path Planning Method for Axial Printing}

\subsection{The Path Planning Algorithm Flow for Axial Printing}

The flow of the path planning algorithm along the axial printing of pipe parts is shown in Figure 5.

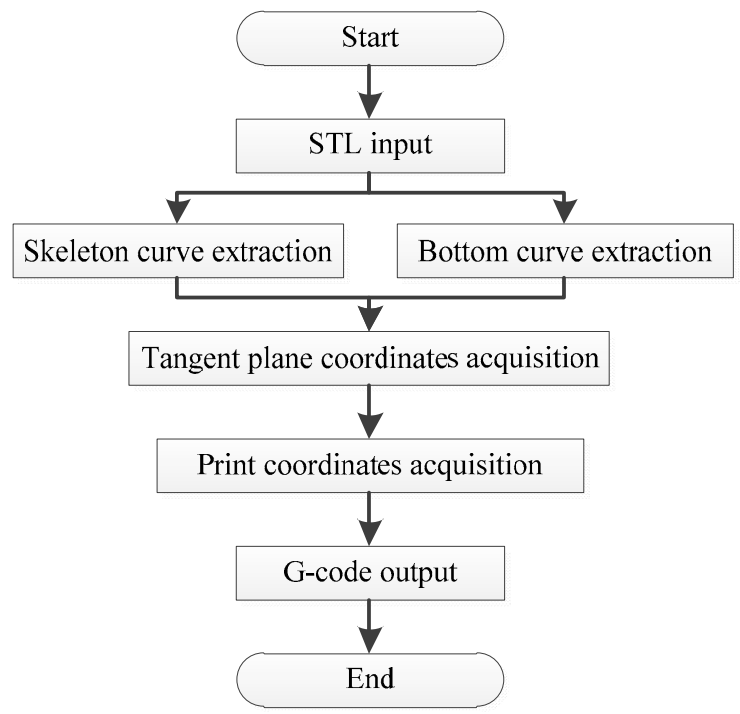

Figure 5. The path planning algorithm flow for axial printing.

The specific implementation steps of the algorithm are as follows.

(1) Input the STL file of the pipe part model, which is modeled and exported by using SolidWorks (Dassault Systèmes, Vélizy-Villacoublay, France).

(2) The skeleton point set of the model is obtained through the STL file of the model. The skeleton point set is fitted to obtain the skeleton curve equation of the model.

(3) The bottom point set of the model is obtained through the STL file of the model. The bottom point set is fitted to obtain the bottom curve equation of the model.

(4) Slice along the direction perpendicular to the tangent vector of the skeleton curve, to obtain the tangent plane of the model, as shown in Figure 6. The coordinate points on the tangent plane of the model are obtained by the skeleton curve equation and the bottom curve equation.

(5) The nozzle direction corresponding to the coordinate point of the model is not along the Z-axis, so the current nozzle direction should be rotated to the direction of the actual printing, and the coordinate point corresponding to the nozzle rotation is the coordinate point of the actual printing.

(6) Obtain the actual printed coordinate points and the rotation angle corresponding to the platform, and output G-code. 


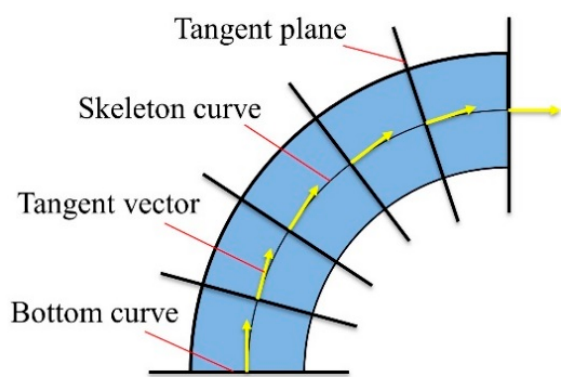

Figure 6. The slicing method of pipe parts model.

\subsection{Skeleton Curve Extraction and Parameter Calculation}

\subsubsection{Skeleton Curve Extraction}

Figure 7 shows the skeleton curve extraction process of the pipe part model.

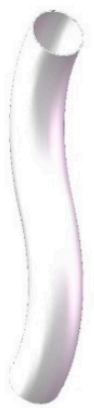

(a)

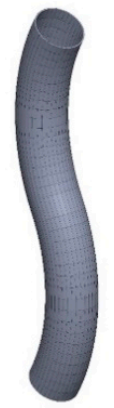

(b)

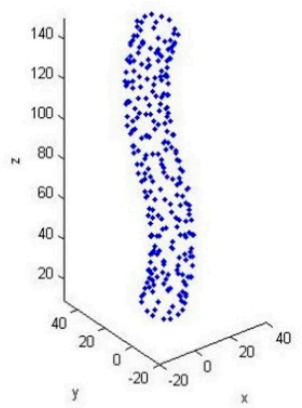

(c)

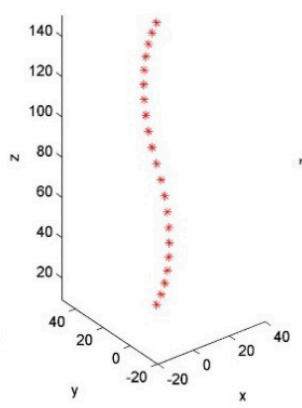

(d)

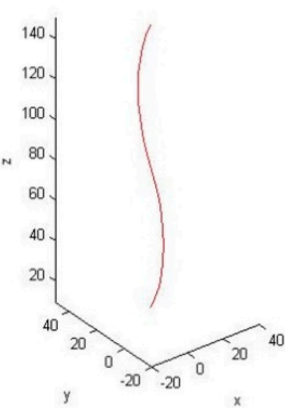

(e)

Figure 7. Skeleton curve extraction process: (a) Designed model, (b) Stereolithography (STL) file, (c) Point cloud, (d) Skeleton point, (e) Skeleton curve.

First, the target model is designed (Figure 7a), and the STL format file is obtained (Figure 7b). Secondly, the point cloud model file (Figure 7c) is extracted from the data of triangular surface in the STL file, and the skeleton point set of the model (Figure 7d) is extracted by the mean curvature flow (MCF) algorithm. Finally, the skeleton point set is fitted by the Bezier curve fitting method, to generate the skeleton curve (Figure 7e). The curve equation generated by fitting is as follows.

$$
S(u)=\left\{\begin{array}{l}
x_{s}(u) \\
y_{s}(u) \\
z_{S}(u)
\end{array} \quad 0 \leq u \leq 1\right.
$$

The curve equation can be adjusted according to the value of $u$. When $u=0$, the coordinate point of the equation is located at the coordinate origin.

\subsubsection{Calculate the Coordinates of the Cross-Section Points and Their Tangent Vectors}

The cross-section point is the intersection point between the tangent plane and the skeleton curve. The model slicing method is to slice along the direction perpendicular to the tangent vector of the skeleton curve. The skeleton curve is segmented, on average, to simplify the calculation. The arc length of the skeleton curve between the tangent planes is the same, and when the number of the tangent planes is more, the accuracy of the printing route is higher. According to the actual accuracy requirements, the arc length of each section is set to $S_{\mathrm{m}}$. According to the integral formula of the arc length, the total arc length $S$ is as follows.

$$
S=\int_{0}^{1} \sqrt{x^{\prime}{ }_{S}(u)^{2}+y^{\prime}{ }_{S}(u)^{2}+z^{\prime}{ }_{S}(u)^{2}} d u
$$


In this paper, the arc length $S$ is calculated by a numerical integration method, then the number of slice layers is LayerNums $=1+S / S_{m}$, and the number of layers is rounded, then the new arc length value is $S_{m}=S /($ LayerNums -1$)$.

From the starting point of the skeleton curve to the $i$ segment, the total arc length value is:

$$
S\left(u_{i}\right)=\int_{0}^{u_{i}} \sqrt{x^{\prime}{ }_{s}(u)^{2}+y^{\prime}{ }_{s}(u)^{2}+z^{\prime}{ }_{s}(u)^{2}} d u=i \times S_{m}
$$

The value of $u_{i}$ can be obtained from Equation (3), so the coordinate of the cross-section point is $P\left(u_{i}\right)=\left(x_{s}\left(u_{i}\right), y_{s}\left(u_{i}\right), z_{s}\left(u_{i}\right)\right)$, so the tangent vector of this point is $\tau_{i}=P\left(u_{i}\right)^{\prime}=$ $\left(x_{s}{ }^{\prime}\left(u_{i}\right), y_{s}{ }^{\prime}\left(u_{i}\right), z_{s}{ }^{\prime}\left(u_{i}\right)\right)$.

\subsection{Bottom Curve Extraction and Parameter Calculation}

\subsubsection{Bottom Curve Extraction}

Figure 8 shows the extraction process of the bottom curve of the pipe part model.

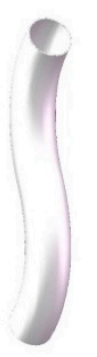

(a)

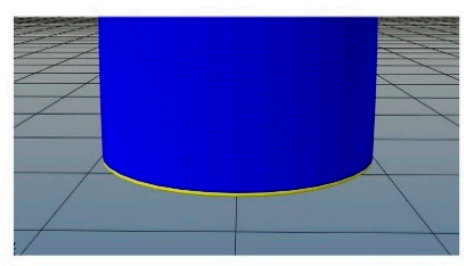

(d)

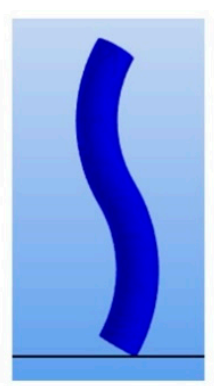

(b)

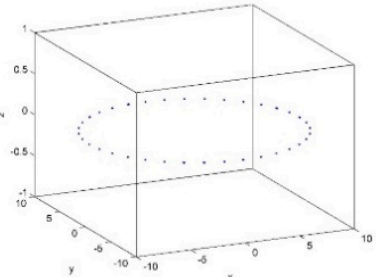

(e)

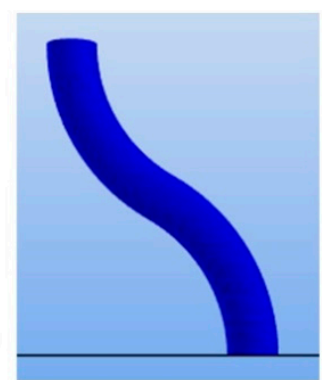

(c)

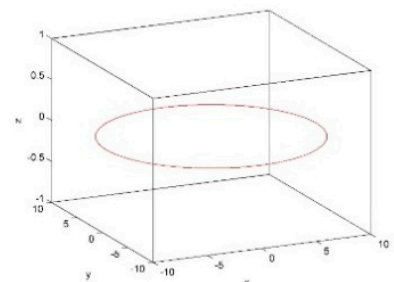

(f)

Figure 8. Bottom curve extraction process: (a) Designed model, (b) STL import, (c) Position adjustment, (d) Model slice, (e) Bottom points, (f) Bottom curve

Import the STL file into the Repetier-Host software (Hot-World GmbH \& Co. KG, Willich, Germany), make the end face of the model coincide with the bottom of the receiving platform by adjusting the position of the model, and obtain the coordinate point set of the contour outside the first layer of the model through the traditional slicing method. By fitting these point sets with MATLAB (The MathWorks, Inc., Natick, MA, USA), the equation of the outer contour of the first layer is obtained, which is called the bottom curve equation. The equation of the bottom curve generated by fitting is as follows.

$$
L(t)=\left\{\begin{array}{l}
x_{\mathcal{c}}(t) \\
y_{c}(t) \\
z_{\mathcal{c}}(t)
\end{array} \quad 0 \leq t<2 \pi\right.
$$

The bottom curve equation is adjusted, so that the center point of the image displayed by the equation is at the origin of coordinates.

Where $z_{c}(t)=0$, and the point $Q(0)=\left(x_{c}(0), y_{c}(0), 0\right)$ is the starting point coordinate of the equation. 


\subsubsection{Calculate the Coordinates of the Bottom Curve Equation}

The position of the coordinate point of the bottom curve equation is the starting point of each path when printing. When printing, the width of each path is the same, denoted by $L_{m}$, according to the arc length integral formula, the arc length $L$ of the bottom curve equation is:

$$
L=\int_{0}^{2 \pi} \sqrt{x_{c}^{\prime}(t)^{2}+y_{c}^{\prime}(t)^{2}} d t
$$

Then, the number of printed paths is PathNums $=L / L_{m}$, and by rounding the number of paths, the new arc length value is $L_{m}=L /$ PathNums. The total arc length value from the starting point of the equation to the $j$ segment is:

$$
L\left(t_{j}\right)=\int_{0}^{t_{j}} \sqrt{x^{\prime}{ }_{c}(t)^{2}+y^{\prime}{ }_{c}(t)^{2}} d t=j \times L_{m}
$$

The value of $t_{j}$ can be obtained from Equation (6), so the coordinate of the $j+1$ point is $Q\left(t_{j}\right)=\left(x_{c}\left(t_{j}\right), y_{c}\left(t_{j}\right), 0\right)$.

Where, $t_{j}$ is the angle between point $Q(0)$ and point $Q\left(t_{j}\right)$ to the origin, as shown in Figure 9.

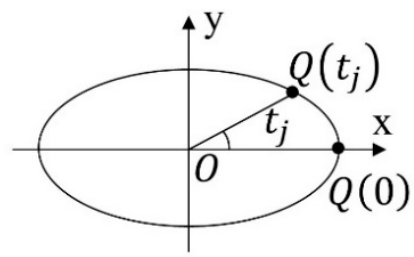

Figure 9. Angle position.

\subsection{Tangent Plane Coordinates Acquisition}

In the case of obtaining the coordinates of the section points of the skeleton curve and its tangent vectors and the coordinates of the bottom curve equation, the coordinate point set on any tangent plane can be obtained by utilizing the coordinate system transformation.

The transformation of the three-dimensional coordinate system, which is essentially the transformation of the origin and the orthogonal basis vectors, is represented by translation and rotation in space. According to the computer graphics theory, two graphs with different positions and the same shape in space can reach the position of another graph through the transformation of the coordinate system. As shown in Figure 10, shape A reaches the position of shape B through rotation, and then the position of shape $\mathrm{C}$ is obtained through translation.
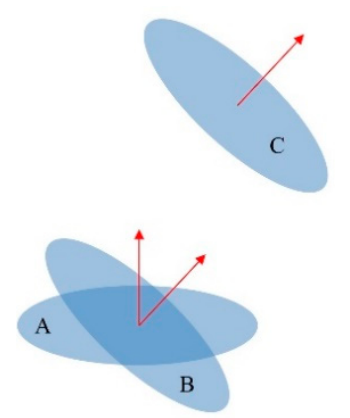

Figure 10. Shape transformation.

The transformation of the position between two shapes can be viewed as the transformation between vectors that are perpendicular to the shape. Figure 11 shows that the vector T1 parallel to the 
Z-axis can be converted into a vector T4 through the transformation of the coordinate system. First, rotate the vector T1 counterclockwise by $\theta$ around the $Y$-axis to obtain the vector T2. Next, rotate the vector T2 counterclockwise by $\delta$ around the Z-axis to obtain the vector T3. Finally, the vector T3 is moved from the origin $O$ to the point $P$, to obtain the vector T4.

Simultaneously, with the vector transformation on the shape, the coordinates of the points on the shape also change correspondingly. Knowing the coordinates of the shape A, the coordinates on the shape $\mathrm{C}$ can be obtained after the coordinate system transformation. Therefore, when the coordinates of the bottom surface are known, the coordinate value of the point set on any tangent plane of the pipe part can be obtained by the method of coordinate system transformation.

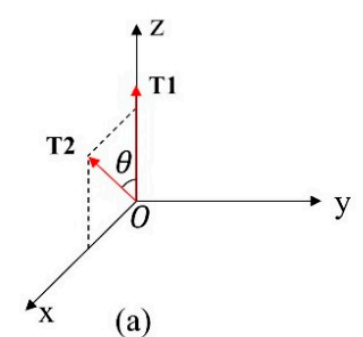

(a)

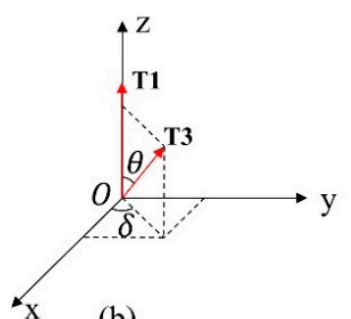

(b)

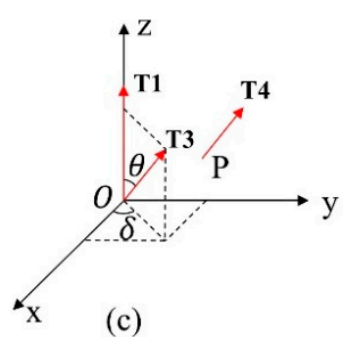

(c)

Figure 11. Vector transformation: (a) $Y$-axis rotation, (b) Z-axis rotation, (c) Translation.

The transformation of the coordinate axis is realized by matrix transformation.

The rotation matrix around the $Y$-axis is:

$$
\operatorname{Rot} Y(u)=\left[\begin{array}{ccc}
\cos u & 0 & -\sin u \\
0 & 1 & 0 \\
\sin u & 0 & \cos u
\end{array}\right]
$$

The rotation matrix around the $Z$-axis is:

$$
\operatorname{Rot} Z(u)=\left[\begin{array}{ccc}
\cos u & \sin u & 0 \\
-\sin u & \cos u & 0 \\
0 & 0 & 1
\end{array}\right]
$$

The moving distance is the distance from the point on the skeleton curve to the origin:

$$
T(u)=\left[\begin{array}{ccc}
1 & 0 & 0 \\
0 & 1 & 0 \\
x_{s}(u) & y_{s}(u) & z_{s}(u)
\end{array}\right]
$$

where, $\theta$ and $\delta$ are calculated as follows.

The tangent vector of the cross-section points on the skeleton curve is:

$$
P\left(u_{i}\right)^{\prime}=\left(x^{\prime}{ }_{s}\left(u_{i}\right), y^{\prime}{ }_{s}\left(u_{i}\right), z^{\prime}{ }_{s}\left(u_{i}\right)\right)
$$

The modulus of the tangent vector at the cross-section point is:

$$
\left|P\left(u_{i}\right)^{\prime}\right|=\sqrt{x^{\prime}{ }_{s}\left(u_{i}\right)^{2}+y_{s}^{\prime}\left(u_{i}\right)^{2}+z_{s}^{\prime}\left(u_{i}\right)^{2}}
$$

The following can be obtained from the geometric relationship:

$$
\theta_{i}=\arccos \frac{z_{s}^{\prime}\left(u_{i}\right)}{\left|P\left(u_{i}\right)^{\prime}\right|}
$$




$$
\delta_{i}=\arctan \frac{y_{s}^{\prime}\left(u_{i}\right)}{x_{s}^{\prime}\left(u_{i}\right)}
$$

The coordinates of the bottom curve are $Q\left(t_{j}\right)=\left(x_{c}\left(t_{j}\right), y_{c}\left(t_{j}\right), z_{c}\left(t_{j}\right)\right)$ and are represented as a matrix as $Q\left(t_{j}\right)=\left[\begin{array}{lll}x_{c}\left(t_{j}\right) & y_{c}\left(t_{j}\right) & z_{c}\left(t_{j}\right)\end{array}\right]$.

Then, the coordinate value of any point on any tangent plane can be expressed in the matrix form as:

$$
Q_{i, j}=Q\left(t_{j}\right) \times \operatorname{Rot} Y\left(\theta_{i}\right) \times \operatorname{RotZ}\left(\delta_{i}\right) \times T\left(u_{i}\right)
$$

where, $Q_{i, j}$ is the matrix form of the coordinate value of the point on the $j+1$ path on the $i+1$ tangent plane.

\subsection{Print Coordinates Acquisition}

Through the transformation of the three-dimensional coordinate system, the coordinate points on any tangent plane are obtained. The direction of the nozzle corresponding to these coordinate points is parallel to the direction of $O-Q\left(t_{j}\right)$, as shown in Figure 12. Where the point $O$ is the center point on the bottom curve, and the point $Q\left(t_{j}\right)$ is the coordinate point on the bottom curve. Simultaneously, the direction of the nozzle is not parallel to the direction of the Z-axis, so the direction of the current nozzle should be rotated to the direction parallel to the Z-axis.

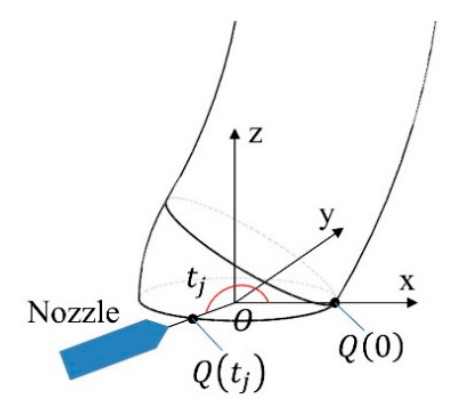

Figure 12. Nozzle direction.

The rotation of the model coordinate points causes the nozzle to rotate. First, rotate the model in Figure 12 clockwise by $90^{\circ}$ around the $Y$-axis, to obtain the position in Figure 13 . Then, rotate the model $t_{j}$ clockwise around the Z-axis, so that the nozzle direction is parallel to the Z-axis. Finally, the entire model is shifted from the position of point $O$ to the position of point $H$. Where point $H$ is the position where the printing support is located, and point $H$ is on the rotation center of $C$-axis.

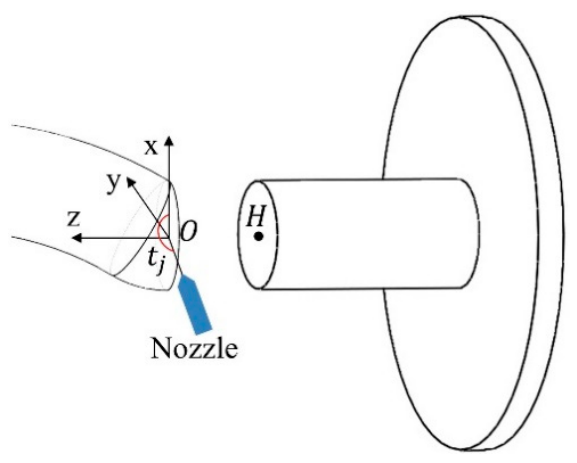

Figure 13. Get print coordinates.

The coordinate value of point $H$ is determined by the structure of the printing platform and the support length. The matrix form of the coordinate value of point $H$ is $H=\left[\begin{array}{ccc}x_{H} & y_{H} & z_{H}\end{array}\right]$. 
The translation matrix of the model is:

$$
T_{H}=\left[\begin{array}{ccc}
1 & 0 & 0 \\
0 & 1 & 0 \\
x_{H} & y_{H} & z_{H}
\end{array}\right]
$$

Therefore, the matrix form of the coordinate value of the point on the printed path can be expressed as:

$$
F_{i, j}=Q_{i, j} \times \operatorname{Rot} Y\left(\frac{\pi}{2}\right) \times \operatorname{Rot} Z\left(t_{j}\right) \times T_{H}
$$

where, $F_{i, j}$ is the matrix form of the coordinate value of the point at which the $j+1$ path is located on the $i+1$ tangent plane.

The points $F_{0, j}, F_{1, j}, \ldots, F_{n, j}$ are connected to form a printing path. Where, $0 \leq j<$ PathNums, and $n$ is the number of slice layers. For each printed path, rotate the $C$-axis by $t_{j}-t_{j-1}$.

\subsection{Path Simulation Results}

Based on the above algorithm, Python was used to write programs and output G-codes. The simulation results of Repetier-Host software were shown in Figure 14.

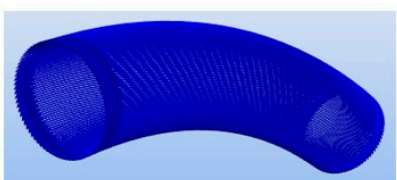

(a1)

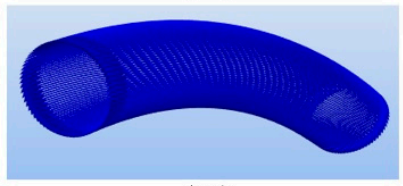

(a2)

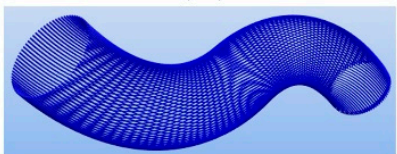

(a3)

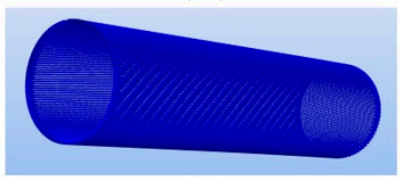

(a4)

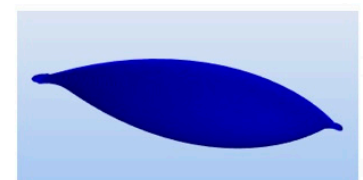

(b1)

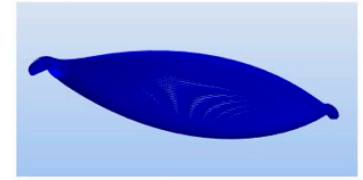

(b2)

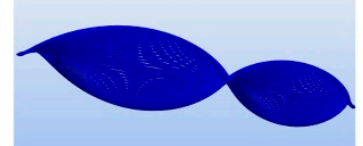

(b3)

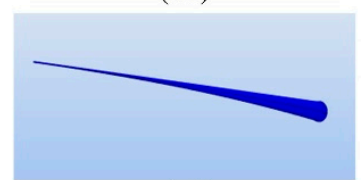

(b4)

Figure 14. Print path simulation results: (a1) Before rotation, circular section and plane single segment curve, (a2) Before rotation, elliptic section and plane single segment curve, (a3) Before rotation, circular section and plane multi-segment curve, (a4) Before rotation, circular section and space curve, (b1) After rotation, circular section and plane single segment curve, (b2) After rotation, elliptic section and plane single segment curve, (b3) After rotation, circular section and plane multi-segment curve, (b4) After rotation, circular section and space curve.

Figure 14 shows the simulation results of the print path. Figure 14a shows the simulation results before the nozzle is rotated, and Figure $14 \mathrm{~b}$ shows the simulation results after the nozzle is rotated, that is, the nozzle's moving path during the actual printing.

The first group of pictures (Figure 14(a1,b1)) represents the simulation results of the circular section shape of the pipe parts, and the skeleton curve of the plane single segment curve. The second group of pictures (Figure 14(a2,b2)) represents the simulation results of the pipe parts with an elliptic section, and a skeleton curve with a plane single segment curve. The third group of pictures (Figure 14(a3,b3)) 
represents the simulation results of the circular section shape of the pipe parts and the skeleton curve of the plane multi-segment curve. The fourth group of pictures (Figure 14(a4,b4)) represents the simulation results of the circular section shape of the pipe parts and the skeleton curve of the space curve.

The simulation results show that the algorithm in this study is not only applicable to the pipe parts whose skeleton curve is a plane curve, but also applicable to the pipe parts whose skeleton curve is spatial. The algorithm of this study has some generality to realize the axial printing along the pipe.

\section{Five-axis 3D Printing Platform Development and Test Verification}

\subsection{Development of a 5-axis 3D Printing Platform}

This paper describes a self-developed 5-axis MEX platform utilized for the printing verification of the algorithm, as shown in Figure 15. The rotation range of $A$-axis is $\pm 90^{\circ}$, and the $C$-axis is equipped with a conductive slip ring, which can be rotated at any angle. The platform adopts ATMEGA 2560 as the main control chip of the lower computer to control the five motion axes and the extruder motor, and uses Marlin Rumba series firmware to communicate with the computer, to realize the multi-axis motion control.

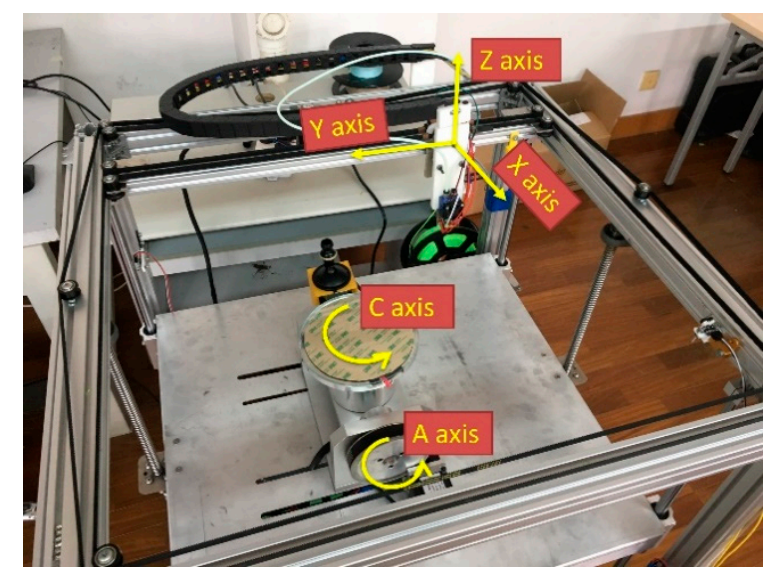

Figure 15. 5-axis 3D printing platform.

The material used for the validation of the algorithm and the developed 5-axis platform was standard PLA filaments from (Rambo Printing Consumables Co., Ltd., Ningbo, China). Three colors (green, red and yellow) were selected, but all of them had similar specifications and processing recommendations, as given by the manufacturer (Table 1).

Table 1. Material specifications and processing recommendations for polylactic acid (PLA) filaments used.

\begin{tabular}{cc}
\hline Specification & Value \\
\hline Density & $1.25 \pm 0.05 \mathrm{~g} / \mathrm{cm}^{3}$ \\
Melt flow index $\left(190^{\circ} \mathrm{C} 2.16 \mathrm{~kg}\right)$ & $5-7 \mathrm{~g} / 10 \mathrm{~min}$ \\
Water absorption & $0.5 \%$ \\
Maximum tensile stress & $\geq 60 \mathrm{MPa}$ \\
Bending modulus & $\geq 60 \mathrm{MPa}$ \\
Elongation at break & $\geq 3.0 \%$ \\
Filament diameter & $1.75 \mathrm{~mm}$ \\
Printing temperature & $195^{\circ} \mathrm{C}$ \\
Hotbed temperature & $50^{\circ} \mathrm{C}$ \\
\hline
\end{tabular}




\subsection{Experimental Results and Analysis}

\subsubsection{Print Result Analysis}

First, the inner surface of the part to be printed is extracted, and the pipe support is printed using the traditional 3D printing method according to the conventional slicing. It should be noted that the support should be in the center of rotation of the platform. Then, rotate $A$-axis by $90^{\circ}$ to place the pipe support horizontally, import G-code outputted by Python, and print it axially. The printing results of a typical sample are shown in Figure 16.

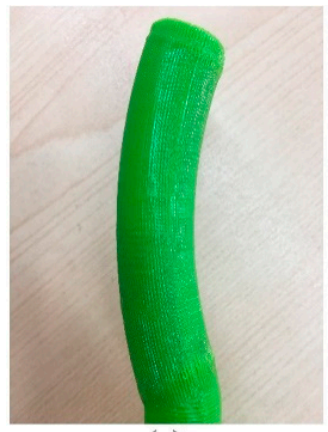

(a)

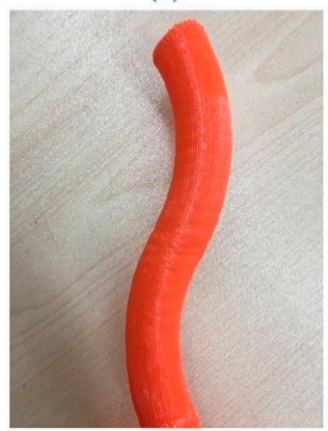

(c)

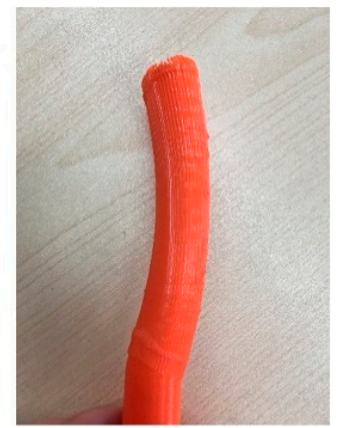

(b)

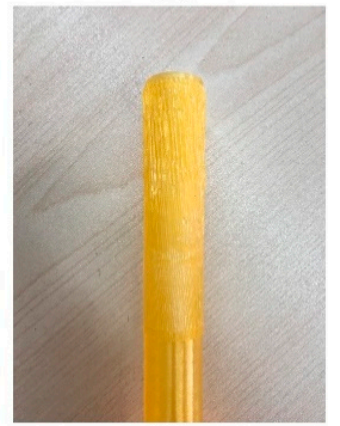

(d)

Figure 16. Typical print sample: (a) Circular section and plane single segment curve, (b) Elliptic section and plane single segment curve, (c) Circular section and plane multi-segment curve, (d) Circular section and space curve.

It can be seen from the printing results in Figure 16 that the G-code outputted by this algorithm can realize the axial printing of pipe parts. This algorithm can realize the printing of pipe parts whose skeleton curve is a plane curve (as shown in Figure 16a-c), as well as the printing of pipe parts whose skeleton curve is a space curve (as shown in Figure 16d). The cross-section of pipe parts can be arbitrarily closed biaxial symmetric graph. In this paper, the cross-section shape of printed pipe parts is circular and elliptic, for example. It should be noted that the curvature of the skeleton curve of pipe parts should not be too large; otherwise, it is easy to cause the collision between the nozzle and the print piece.

\subsubsection{Mechanical Properties Test}

\section{Test Samples and Experimental Design}

Mechanical tests were carried out to verify the effect of axial printing on the mechanical properties of pipe parts. Since there is no standard for the direct testing of pipe parts, the test samples were generated by simulating the axial printing path of pipe parts, and the mechanical testing experiments were conducted by using the plastic tensile property testing standard GB/T 1040.2-2006/ISO 527-2:1993, and the plastic bending property testing standard GB/T 9341-2008/ISO 178:2001. The printing material was PLA with the characteristics given in Table 1, and the testing machine was TY8000 series material 
testing machine (Tianyuan Test Equipment Co., Ltd., Yangzhou, China). The size of the test sample is shown in Figure 17.

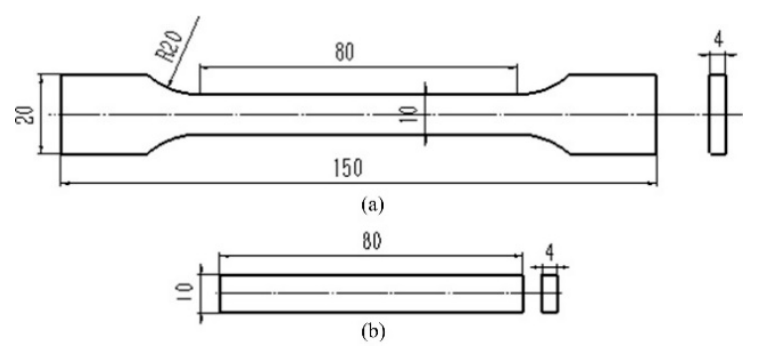

Figure 17. Test sample size: (a) Tensile sample, (b) Three-point bending sample.

Figure $17 \mathrm{a}$ is the standard sample for the tensile test, with a standard spacing of $80 \mathrm{~mm}$, and Figure $17 \mathrm{~b}$ is the standard sample for the three-point bending test, with a span of $50 \mathrm{~mm}$.

The thickness of the sample is $4 \mathrm{~mm}$, and the thickness of the layer is $0.2 \mathrm{~mm}$, which makes a total of 20 layers. To study the influence of axial printing on the mechanical properties of printed specimens, the number of layers printed along the radial path of the sample was determined according to the thickness of the actual supporting pipe, and the number of layers printed along the axial path of the sample was determined according to the thickness of the axial strengthening printing, as shown in Figure 18.

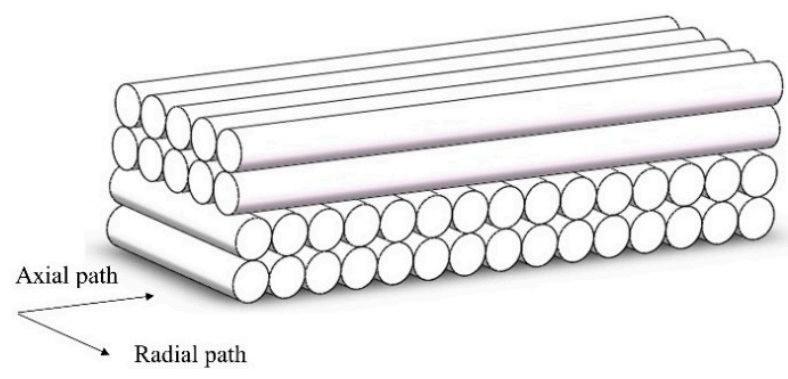

Figure 18. The path direction.

To study the enhancement rule of the axial path on the mechanical properties, the radial path is gradually replaced with an axial path, and a set of samples is printed for every five layers replaced, that is, the number of axial path layers is $0,5,10,15,20$ layers respectively, with five samples for each group. The samples with an axial path of zero layers were used as the control group, while the remaining samples were used as the experimental group. Figure 19 shows a localized view of a printed specimen. The lower part is the radial path of 10 layers, and the upper part is the axial path of 10 layers.

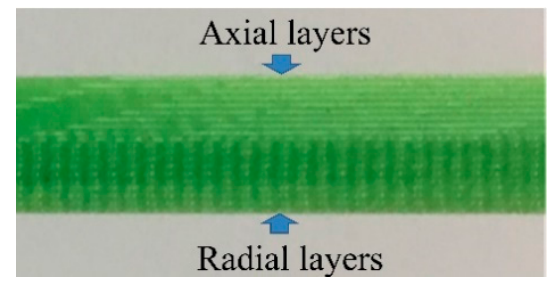

Figure 19. Local diagram of the experimental sample.

The Tensile Test

The standard sample was tensile tested at a rate of $2 \mathrm{~mm} / \mathrm{min}$, as shown in Figure 20. 


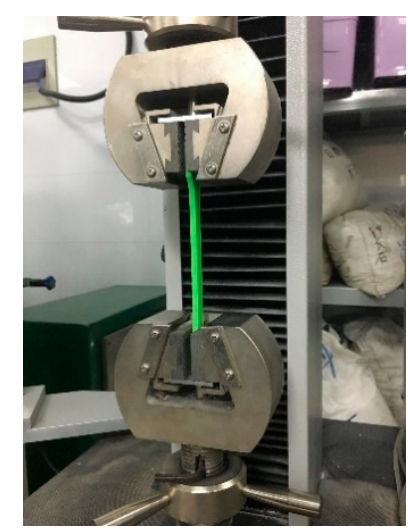

Figure 20. The tensile test.

The stress-strain curve of the tensile test is shown in Figure 21.

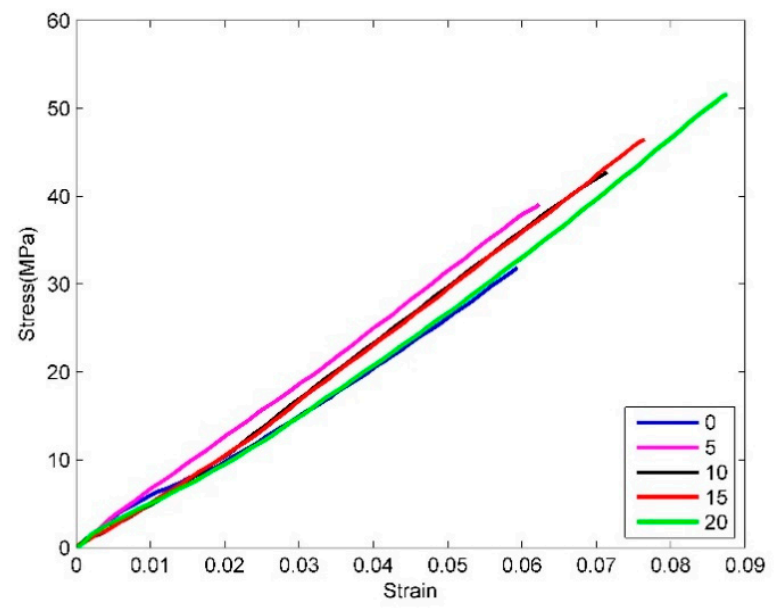

Figure 21. Tensile test stress-strain curve.

The average maximum tensile stress of the same set of samples, shown in Table 2. Standard deviation is depicted in brackets.

Table 2. Tensile test the maximum stress of each group.

\begin{tabular}{cc}
\hline No. of Axial Layers & Maximum Tensile Stress (MPa) \\
\hline 0 & $34.1(3.8)$ \\
5 & $39.0(2.6)$ \\
10 & $42.4(1.0)$ \\
15 & $47.2(2.8)$ \\
20 & $51.6(1.5)$ \\
\hline
\end{tabular}

The data in Table 2 are represented by a graph, as shown in Figure 22. The horizontal axis represents the number of layers printed in the axial direction, and the vertical axis represents the maximum stress value of the tensile experiment. Increasing the axial layers and the decreasing radial layers, the tensile stress of the print increases by an average of $2.6 \%$ for each additional layer of axial layers, which proves that the axial printing does have an enhancement effect, and the enhancement effect is also improved with the increase of the number of reinforcement layers. 


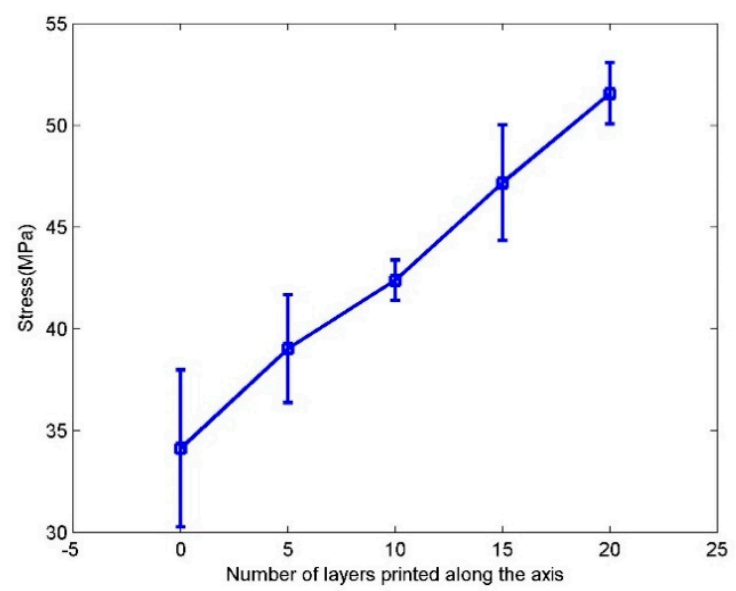

Figure 22. Tensile test the maximum stress of each group.

It is essential to point out that, even when all the layers are in the axial direction, the maximum tensile stress given in the material specifications (Table 1) is not reached. These low tensile properties indicate the presence of voids between the deposited layers. The current algorithm does not solve this issue, but this should be investigated in future studies.

\section{Three-Point Bending Test}

In the three-point bending experiment, the downward part of the printed piece is stretched, and the upward part is compressed. When the downward part of the printed piece is stretched, stress concentration is likely to occur. The purpose of this experiment is to verify that the axial path reduces the influence of stress concentration, thus enhancing the mechanical properties of the printed piece. Therefore, in the three-point bending experiment, the axial path of the sample piece is downward, while the radial path is upward.

Three-point bending test was performed on standard specimens (Figure 17b), and the compression speed was $2 \mathrm{~mm} / \mathrm{min}$. The experimental set up is shown in Figure 23.

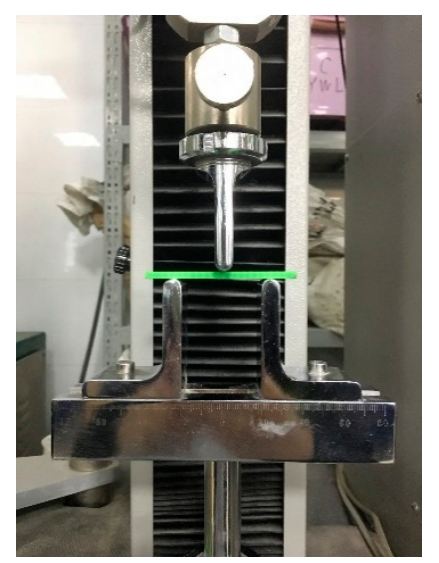

Figure 23. Three-point bending test.

The stress-strain curve of the three-point bending test is shown in Figure 24. 


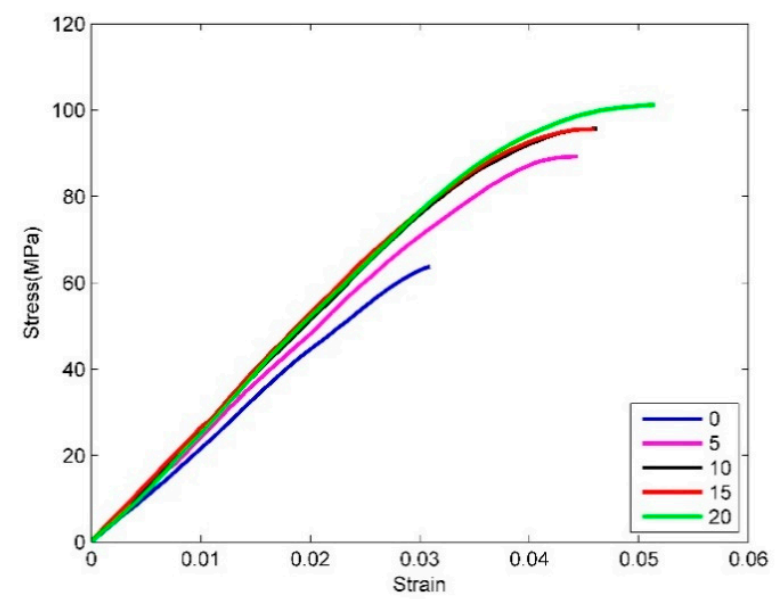

Figure 24. Representative three-point bending test stress-strain curve.

The average maximum bending stress of the same set of samples are shown in Table 3. Standard deviation is depicted in brackets.

The data in Table 3 are represented by a graph, as shown in Figure 25. It can be seen from the experimental results that the strength of the printed pieces with 5 axial layers is $38.5 \%$ higher than that with 0 axial layers. However, as the number of axial layers continues to increase, the strength of the printed pieces does not increase significantly. Therefore, axial printing can reduce the impact of stress concentration on the printed piece, thus enhancing the mechanical properties of the printed samples.

Table 3. Three-point bending tests the maximum stress of each group.

\begin{tabular}{cc}
\hline No. of Axial Layers & Maximum Bending Stress (MPa) \\
\hline 0 & $64.4(2.5)$ \\
5 & $89.2(1.7)$ \\
10 & $95.9(1.3)$ \\
15 & $95.1(1.4)$ \\
20 & $102.0(1.8)$ \\
\hline
\end{tabular}

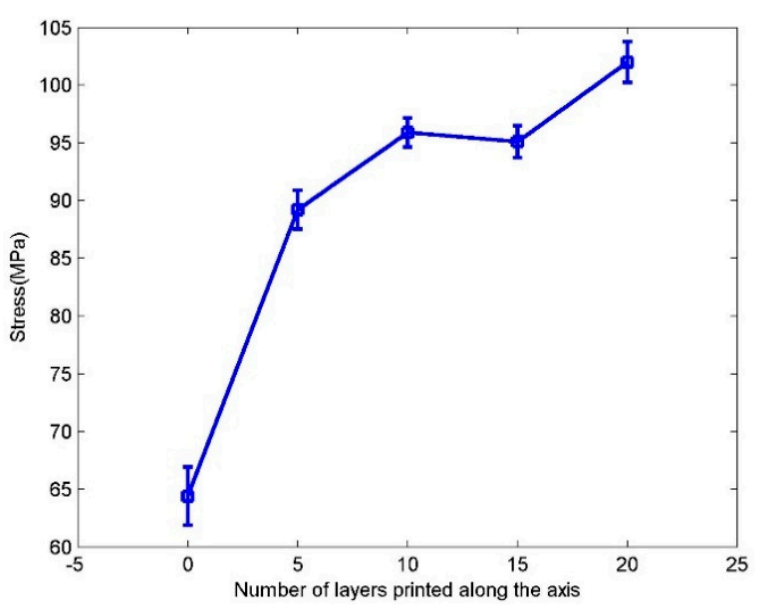

Figure 25. Three-point bending test of the maximum stress of each group.

By simulating the path of axial printing to carry out the mechanical test; although it cannot wholly reflect the real situation of the force on pipe parts, it can show to some extent that axial printing can improve the mechanical properties of the printed parts. 


\section{Conclusions}

Given the weak mechanical properties of MEX parts along the Z-axis, this paper proposes the method of axial printing along pipe parts to enhance their mechanical properties. The neutral skeleton curve equation and the bottom curve equation of the model are obtained through the STL file derived from the model. The coordinates on any tangent plane are obtained through the transformation of the coordinate axis, and the coordinate points on each tangent plane are connected, that is the printing path. The axial printing algorithm is implemented through a self-developed 5-axis printing platform. It is shown that axial printing can improve the mechanical properties of PLA printed specimens by tensile and three-point bending tests.

The algorithm proposed in this paper is adequate for pipe parts whose section shape is biaxially symmetric, but it is not applicable for those whose section shape axisymmetric. For pipe parts with a more pronounced skeleton curvature, a collision between the nozzle and the print may occur during the fabrication process. This paper does not put forward the judgment condition of collision. In the process of multi-layer printing, there are gaps between layers, and this algorithm does not implement gap filling, that can substantially improve the mechanical properties of the printed parts. Future work should revolve around these questions.

Author Contributions: Conceptualization, M.A. and H.L.; Funding acquisition, Q.H. and H.L.; Investigation, W.Z.; Methodology, H.Z. and W.Z.; Software, W.Z.; Supervision, Q.H.; Writing—original draft, W.Z.; Writing一review and editing, H.Z. and J.G.-G. All authors have read and agreed to the published version of the manuscript.

Funding: The authors acknowledge funding support from the National Natural Science Foundation of China (Grant 51775324 and 51375292) and the China Scholarship Council (No. 201806895016) and the Shanghai Key Laboratory of Intelligent Manufacturing and Robotics (Grant No. ZK1304). The research work was supported by the fund of Austrian-Chinese Cooperative R\&D Projects (FFG No.860384).

Conflicts of Interest: The authors declare no conflict of interest.

\section{References}

1. Tay, Y.W.D.; Panda, B.; Paul, S.C.; Noor Mohamed, N.A.; Tan, M.J.; Leong, K.F. 3D printing trends in building and construction industry: A review. Virtual Phys. Prototyp. 2017, 12, 261-276. [CrossRef]

2. Goyanes, A.; Fina, F.; Martorana, A.; Sedough, D.; Gaisford, S.; Basit, A.W. Development of modified release 3D printed tablets (printlets) with pharmaceutical excipients using additive manufacturing. Int. J. Pharm. 2017, 527, 21-30. [CrossRef]

3. Jonusauskas, L.; Gailevicius, D.; Mikoliunaite, L.; Sakalauskas, D.; Sakirzanovas, S.; Juodkazis, S.; Malinauskas, M. Optically Clear and Resilient Free-Form micro-Optics 3D-Printed via Ultrafast Laser Lithography. Materials (Basel) 2017, 10, 12. [CrossRef]

4. Jin, Y.; Du, J.; He, Y.; Fu, G. Modeling and process planning for curved layer fused deposition. Int. J. Adv. Manuf. Technol. 2016, 91, 273-285. [CrossRef]

5. Isa, M.A.; Lazoglu, I. Five-axis additive manufacturing of freeform models through buildup of transition layers. J. Manuf. Syst. 2019, 50, 69-80. [CrossRef]

6. Ding, D.; Pan, Z.; Cuiuri, D.; Li, H. A practical path planning methodology for wire and arc additive manufacturing of thin-walled structures. Robot. Comput. Integr. Manuf. 2015, 34, 8-19. [CrossRef]

7. Feng, J.; Fu, J.; Lin, Z.; Shang, C.; Li, B.; Campbell, R.I.; Gibson, I. Direct slicing of T-spline surfaces for additive manufacturing. Rapid Prototyp. J. 2018. [CrossRef]

8. Xie, F.; Chen, L.; Li, Z.; Tang, K. Path smoothing and feed rate planning for robotic curved layer additive manufacturing. Robot. Comput. Integr. Manuf. 2020, 65. [CrossRef]

9. Lee, K.; Jee, H. Slicing algorithms for multi-axis 3-D metal printing of overhangs. J. Mech. Sci. Technol. 2015, 29, 5139-5144. [CrossRef]

10. Ding, D.; Pan, Z.; Cuiuri, D.; Li, H.; Larkin, N.; van Duin, S. Automatic multi-direction slicing algorithms for wire based additive manufacturing. Robot. Comput. Integr. Manuf. 2016, 37, 139-150. [CrossRef]

11. Xu, K.; Chen, L.; Tang, K. Support-Free Layered Process Planning Toward 3 + 2-Axis Additive Manufacturing. IEEE Trans. Autom. Sci. Eng. 2019, 16, 838-850. [CrossRef] 
12. Wu, R.; Peng, H.; Guimbretière, F.; Marschner, S. Printing arbitrary meshes with a 5DOF wireframe printer. ACM Trans. Graph. 2016, 35, 1-9. [CrossRef]

13. Zhao, G.; Ma, G.; Feng, J.; Xiao, W. Nonplanar slicing and path generation methods for robotic additive manufacturing. Int. J. Adv. Manuf. Technol. 2018, 96, 3149-3159. [CrossRef]

14. Xu, K.; Li, Y.; Chen, L.; Tang, K. Curved layer based process planning for multi-axis volume printing of freeform parts. Comput.-Aided Des. 2019, 114, 51-63. [CrossRef]

15. Dai, C.; Wang, C.C.L.; Wu, C.; Lefebvre, S.; Fang, G.; Liu, Y.-J. Support-free volume printing by multi-axis motion. ACM Trans. Graph. 2018, 37,1-14. [CrossRef]

16. Khan, J.A.; Wu, C.; Liu, Y.J.; Ying, H.; Wang, C.C.L. Interactive Partitioning of 3D Models into Printable Parts. IEEE Comput. Graph. Appl. 2018, 38, 38-53. [CrossRef]

17. Zhao, H.-m.; He, Y.; Fu, J.-z.; Qiu, J.-j. Inclined layer printing for fused deposition modeling without assisted supporting structure. Robot. Comput. Integr. Manuf. 2018, 51, 1-13. [CrossRef]

18. Dickson, A.N.; Ross, K.-A.; Dowling, D.P. Additive manufacturing of woven carbon fibre polymer composites. Compos. Struct. 2018, 206, 637-643. [CrossRef]

19. Sugiyama, K.; Matsuzaki, R.; Ueda, M.; Todoroki, A.; Hirano, Y. 3D printing of composite sandwich structures using continuous carbon fiber and fiber tension. Compos. Part A Appl. Sci. Manuf. 2018, 113, 114-121. [CrossRef]

20. Bin Ishak, I.; Fleming, D.; Larochelle, P. Multiplane fused deposition modeling: A study of tensile strength. Mech. Based Des. Struct. Mach. 2019, 47, 583-598. [CrossRef]

21. Huang, B.; Singamneni, S.B. Curved Layer Adaptive Slicing (CLAS) for fused deposition modelling. Rapid Prototyp. J. 2015, 21, 354-367. [CrossRef]

22. Lim, S.; Buswell, R.A.; Valentine, P.J.; Piker, D.; Austin, S.A.; De Kestelier, X. Modelling curved-layered printing paths for fabricating large-scale construction components. Addit. Manuf. 2016, 12, 216-230. [CrossRef]

23. Tam, K.-M.M.; Mueller, C.T. Additive Manufacturing Along Principal Stress Lines. 3D Print. Addit. Manuf. 2017, 4, 63-81. [CrossRef]

24. Xia, L.; Lin, S.; Ma, G. Stress-based tool-path planning methodology for fused filament fabrication. Addit. Manuf. 2020, 32. [CrossRef]

(C) 2020 by the authors. Licensee MDPI, Basel, Switzerland. This article is an open access article distributed under the terms and conditions of the Creative Commons Attribution (CC BY) license (http://creativecommons.org/licenses/by/4.0/). 